\title{
Sub-TeV hadronic interaction model differences and their impact on air showers
}

\author{
Á. Pastor-Gutiérrez, ${ }^{a, *}$ H. Schoorlemmer, ${ }^{a}$ R.D. Parsons ${ }^{a, b}$ and M.Schmelling ${ }^{a}$ \\ ${ }^{a}$ Max-Planck-Institut für Kernphysik P.O. Box 103980, D 69029, Heidelberg, Germany \\ ${ }^{b}$ Institut für Physik Humboldt-Universität zu Berlin Newtonstr. 15,D 12489, Berlin, Germany \\ E-mail: alvaro.pastor@mpi-hd.mpg.de, harmscho@mpi-hd.mpg.de, \\ daniel.parsons@physik.hu-berlin.de, michael.schmelling@mpi-hd.mpg.de
}

In the sub-TeV regime, the most widely used hadronic interaction models disagree significantly in their predictions for post-first interaction and ground-level particle spectra from cosmic ray induced air showers. These differences generate an important source of systematic uncertainty in their experimental use. We investigate the nature and impact of model uncertainties through a simultaneous analysis of ground level particles and first interaction scenarios. We focus on air shower primaries with energies close to the transition between high and low energy hadronic interaction models, where the dissimilarities have been shown to be the largest and well within the range of accelerator measurements. Interaction models are shown to diverge as several shower scenarios are compared, reflecting intrinsic differences in the model theoretical frameworks.

Additionally, we discuss the importance of interactions in the energy regime where the switching between models occurs $(<1 \mathrm{TeV})$ and the effect of the choice of model on the number of hadronic interactions within cosmic ray induced air showers of higher energies.

$37^{\text {th }}$ International Cosmic Ray Conference (ICRC 2021)

July 12th - 23rd, 2021

Online - Berlin, Germany

\footnotetext{
${ }^{*}$ Presenter
} 


\section{Introduction and setup}

This proceedings is a summary of our recently published work [1]. In a previous study [2], it was found that for cosmic-ray protons with an energy just above the typical switching energy, the properties of the simulated extensive air showers have a strong dependency on the selection of the hadronic interaction model. Severe disagreements were observed which reduced by increasing the primary's energy, reaching a reasonable agreement at energies $>10 \mathrm{TeV}$. In [1], we focus on the low energy domain (close to the transition energy between high and low energy models, $80 \mathrm{GeV}$ ), where the differences between the models are most prominently exposed (up to 60\% difference in the ground level observables).

In order to find the source of such differences, we performed simulations for EPOS-LHC [3], QGSJetII-04 [4], SIBYLL 2.3c [5, 6] and UrQMD [7] hadronic models using the Monte Carlo air shower event generator CORSIKA v7.64 [8]. We compare in detail the nature of the early shower development and its relation to ground-level observables by simultaneously analyzing, event-by-event, the final state immediately after the first interaction $(1 \mathrm{~cm}$ below the interaction point) and at ground level (at $4100 \mathrm{~m}$, HAWC gamma-ray observatory altitude [9]). We used the lateral distribution functions (LDF) of the muon and electromagnetic (EM) components as the main observables at ground level. The different events were characterized by the leading particle type and fraction of shower energy carried, represented by the inelasticity of the collision

$$
\kappa=1-\frac{E_{\mathrm{LP}}}{E_{\mathrm{FI}}}
$$

where $E_{\mathrm{FI}}$ is the total shower energy (sum of all particle energies) after the first interaction and $E_{\mathrm{LP}}$ the leading particle energy.

\section{Simultaneous analysis of the first interaction and ground level products}

As shown in Figure 1 we looked at the ground level contributions from specific first interaction scenarios. We found that for more particular physical events, the models differences in the ground level muons and EM component enlarged. For example, in events lead by muonic particles $\left(\mu^{+}\right.$, $\mu^{-}, \pi^{+}, \pi^{-}, K_{L}^{0}, K^{+}, K^{-}$and $\left.K_{S}^{0}\right)$, QGSJetII-04 produced around 2 times more muons at short core distances $(r<200 \mathrm{~m})$, as shown in Figure 3 in [1].

To disentangle the origin of such differences, we concentrated our analysis on the first interaction. The rates of events as a function of the inelasticity of the leading particle, as shown in Figure 2, provided a normalization-based argument for large part of the disagreement. Nevertheless, the models still differed in the number of muons per event and their distribution along the ground level plane. The multiplicity of muonic particles in the first interaction and their transverse momenta provided sufficient arguments for the observed ground level muon disagreement.

Additionally we studied the proton-air cross-sections implemented in each of the models. While the high energy models reasonably agree, UrQMD shows larger values. In free first interaction simulations, this effect is remarkable. A discussion is presented on Appendix A of [1] based on comparisons to first simulations in [2].

Lastly, in [1] we show how the event generators diverge by studying the number of hadronic interactions in showers initiated at high energies. Large differences have been spotted remarking 

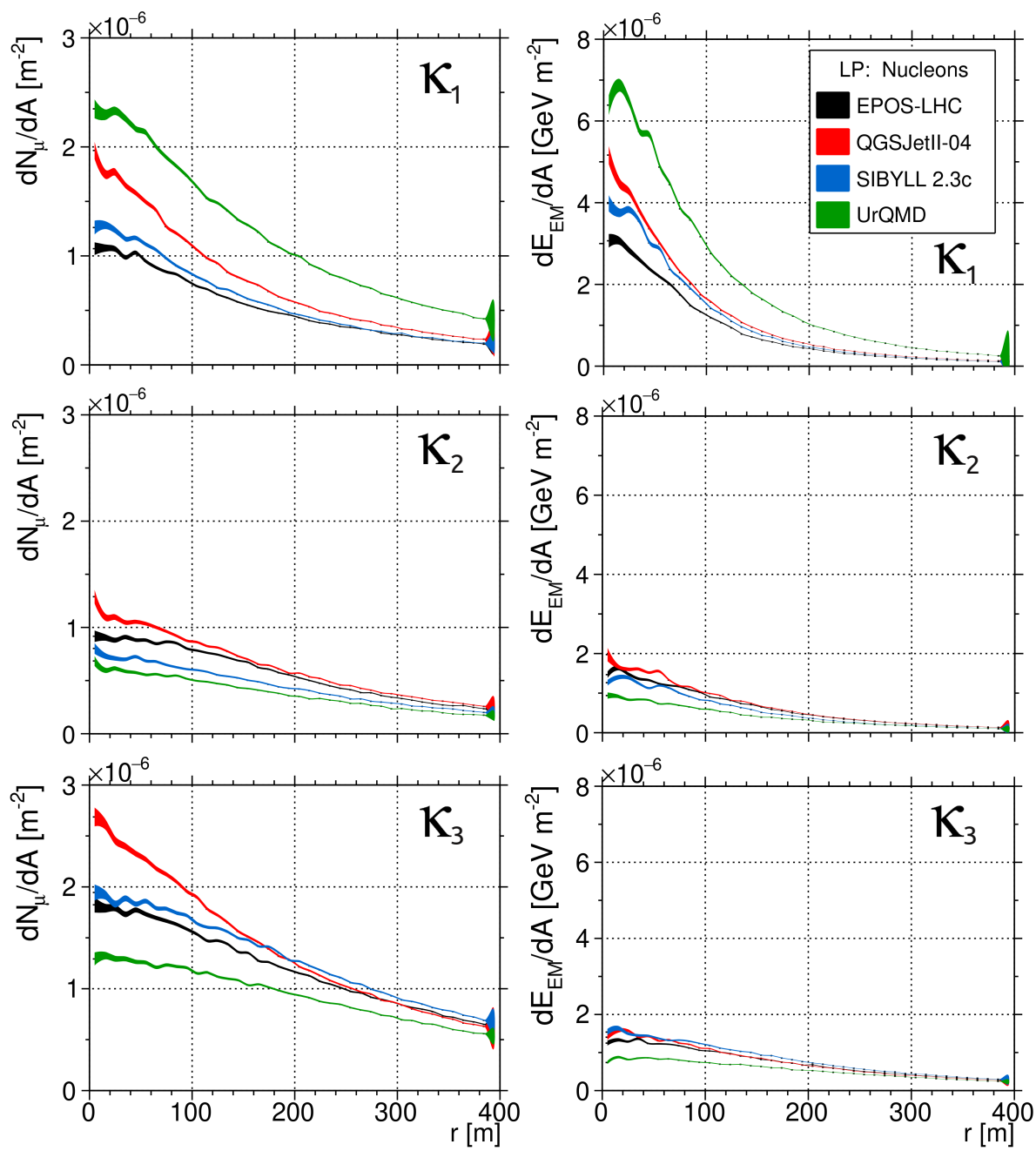

Figure 1: Breakdown of the muon (left panels) and EM component (right panels) LDFs into the contributions from the three inelasticity regimes in nucleon led events. To have leading particles with similar properties, here energy ranges are taken as a proxy for the respective $\kappa$-regions, the boundaries of which are smeared due to event-by-event fluctuations in the energy violation of the models.

how the models produce very dissimilar showers from the same initial conditions. In this line, we have shown how sensitive the shower development is to modifications of the transition energies. The low energy model shows a clear domination of the shower (ruling over $85 \%$ of the interactions in a PeV shower with default transition energy and increasing percentage for higher primaries) while the high energy models set the initial conditions. This points to low energy model investigations as a possible important contribution to the solution of the hadronic interaction model puzzles [10-12].

\section{Summary and conclusions}

Through a detailed investigation of events initiated by $100 \mathrm{GeV}$ protons we cast light on the sources of disagreement between hadronic interaction models [2]. By a simultaneous analysis of the first interaction and ground level particles, we can conclude that models present an intrinsically 




Figure 2: Inelasticity distributions for the four studied models, for events initiated by the two dominant families; nucleons and muonic particles.

different behavior in the low energy regime. An outline on each model's arguments is presented in section 5 of [1].

Phenomenological variables were used to correlate differences in ground level observables to properties of the first interaction for some commonly employed high energy models. Discrepancies in the ground level particle number emerged from event rate deviations that were drawn from the study of first interaction inelasticity distributions. Within same events types (same leading particle and inelasticity), the production of accompanying particles (eg. muonic family particles) disagrees causing differences in the ground-level muon component. The transverse momentum spectra of muonic particles that are produced in the first interaction has also been shown to differ between models. This disagreement becomes worse as their energy increases.

As the focused energy regime falls within the validity range of all models, a reasonable agreement would be expected between all high energy models and UrQMD. The large differences found demand for a convergent model tuning to existing and/or future accelerator data or a redefinition of the transition regime from high to low energy hadronic interaction models that ensures consistency with the high energy partners.

\section{References}

[1] Á. Pastor-Gutiérrez, H. Schoorlemmer, R. D. Parsons, and M. Schmelling. Sub-TeV hadronic interaction model differences and their impact on air showers. Eur. Phys. J. C, 81:369, 2021.

[2] R.D. Parsons and H. Schoorlemmer. Systematic Differences due to High Energy Hadronic 
Interaction Models in Air Shower Simulations in the $100 \mathrm{GeV}-100 \mathrm{TeV}$ Range. Phys. Rev. D, 100(2):023010, 2019.

[3] T. Pierog, Iu. Karpenko, J. M. Katzy, E. Yatsenko, and K. Werner. Epos lhc: Test of collective hadronization with data measured at the cern large hadron collider. Phys. Rev. C, 92:034906, Sep 2015.

[4] S. Ostapchenko. Monte carlo treatment of hadronic interactions in enhanced pomeron scheme: Qgsjet-ii model. Phys. Rev. D, 83:014018, Jan 2011.

[5] F. Riehn, H. Dembinski, R. Engel, A. Fedynitch, Gaisser T.K., and T. Stanev. The hadronic interaction model Sibyll 2.3c and Feynman scaling. In 35th International Cosmic Ray Conference, PoS(ICRC2017)301, July 2017.

[6] Felix Riehn, Ralph Engel, Anatoli Fedynitch, Thomas K. Gaisser, and Todor Stanev. Hadronic interaction model sibyll 2.3d and extensive air showers. Physical Review D, 102(6), Sep 2020.

[7] S.A. Bass, M. Belkacem, M. Bleicher, M. Brandstetter, L. Bravina, C. Ernst, L. Gerland, M. Hofmann, S. Hofmann, J. Konopka, G. Mao, L. Neise, S. Soff, C. Spieles, H. Weber, L.A. Winckelmann, H. Stöcker, W. Greiner, Ch. Hartnack, J. Aichelin, and N. Amelin. Microscopic models for ultrarelativistic heavy ion collisions. Progress in Particle and Nuclear Physics, 41:255 - 369, 1998.

[8] D. Heck, J. Knapp, J. N. Capdevielle, G. Schatz, and T. Thouw. CORSIKA: a Monte Carlo code to simulate extensive air showers. Forschungszentrum Karlsruhe GmbH, February 1998.

[9] A. U. Abeysekara et al. Observation of the crab nebula with the HAWC gamma-ray observatory. The Astrophysical Journal, 843(1):39, 2017.

[10] Alexander Aab et al. Testing Hadronic Interactions at Ultrahigh Energies with Air Showers Measured by the Pierre Auger Observatory. Phys. Rev. Lett., 117(19):192001, 2016.

[11] Sebastian Baur, Hans Dembinski, Matias Perlin, Tanguy Pierog, Ralf Ulrich, and Klaus Werner. Core-corona effect in hadron collisions and muon production in air showers. 22019.

[12] H. P. Dembinski et al. Report on Tests and Measurements of Hadronic Interaction Properties with Air Showers. EPJ Web Conf., 210:02004, 2019. 